\title{
Inhibiting CXCL12 blocks fibrocyte migration and differentiation and attenuates bronchiolitis obliterans in a murine heterotopic tracheal transplant model
}

\author{
David A. Harris, BS, ${ }^{\mathrm{a}}$ Yunge Zhao, MD, PhD, ${ }^{\mathrm{a}}$ Damien J. LaPar, MD, MSc, ${ }^{\mathrm{a}}$ Abbas Emaminia, MD, ${ }^{\mathrm{a}}$ \\ John F. Steidle, BA, ${ }^{a}$ Mark Stoler, MD, ${ }^{b}$ Joel Linden, MD, ${ }^{\mathrm{c}}$ Irving L. Kron, MD, ${ }^{a}$ and Christine L. Lau, MD ${ }^{\mathrm{a}}$
}

\begin{abstract}
Objectives: Fibrocytes are integral in the development of fibroproliferative disease after lung transplantation. Undifferentiated fibrocytes (CD45+anti-collagen 1+CXCR4+) preferentially traffic by way of the CXCR4/ CXCL12 axis and differentiate into smooth muscle actin-producing (CD45+CXCR4 $+\alpha$-smooth muscle actin + ) cells. We postulated that an antibody directed against CXCL12 would attenuate fibrocyte migration and fibro-obliteration of heterotopic tracheal transplant allografts.
\end{abstract}

\begin{abstract}
Methods: A total alloantigenic mismatch murine heterotopic tracheal transplant model of obliterative bronchiolitis was used. The mice were treated with either goat-anti-human CXCL12 $F\left(a^{\prime}\right)_{2}$ or goat IgG F( $\left(\mathrm{b}^{\prime}\right)_{2}$. Buffy coat, bone marrow, and trachea allografts were collected and analyzed using flow cytometry. Tracheal luminal obliteration was assessed using hematoxylin-eosin and Direct Red 80 collagen stain.
\end{abstract}

Results: Compared with the controls, the anti-CXCL12-treated mice showed a significant decrease in tracheal allograft fibrocyte populations at 7 and 21 days after transplantation. Bone marrow and buffy coat aspirates showed the same trend at 7 days. In the anti-CXCL12-treated mice, there was a 35\% decrease in luminal obliteration at 21 days $(65 \%$ vs $100 \%$ obliterated; interquartile range, $38 \%$ vs $10 \% ; P=.010)$ and decreased luminal collagen deposition at 21 and 28 days after transplantation $(P=.042$ and $P=.012$, respectively).

Conclusions: Understanding the role of fibrocytes in airway fibrosis after lung transplantation could lead to a paradigm shift in treatment strategy. Anti-CXCL12 antibody afforded protection against infiltrating fibrocytes and reduced the deterioration of the tracheal allografts. Thus, the CXCR4/CXCL12 axis is a novel target for the treatment of fibro-obliteration after lung transplantation, and the quantification of fibrocyte populations could provide clinicians with a biomarker of fibrosis, allowing individualized drug therapy. (J Thorac Cardiovasc Surg 2013;145:854-61)

Bronchiolitis obliterans syndrome (BOS) is a leading cause of morbidity and mortality after lung transplantation. ${ }^{1}$ Lung fibroblasts and myofibroblasts are critical for the development of fibrosis and are thought to arise from 3 locations: the resident proliferation of fibroblasts, epithelial to mesenchyme transition, and bone marrow-derived mesenchymal progenitor cells, fibrocytes. ${ }^{2}$

Fibrocytes (CD45+anti-collagen 1 [Col1]+CXCR4+) are bone marrow-derived mesenchymal stem cells that are released into the circulation in response to numerous

From the Departments of Surgery ${ }^{\mathrm{a}}$ and Pathology, ${ }^{\mathrm{b}}$ University of Virginia Health System, Charlottesville, Va; and La Jolla Institute for Allergy and Immunology, ${ }^{\mathrm{c}} \mathrm{La}$ Jolla, Calif.

C. L. Lau was supported by a grant sponsored by the National Heart, Lung, and Blood Institute (grant 1K08HL094704-01) and the CVRC Partner's Grant and was the AATS John W. Kirklin Research Fellow.

Disclosures: Authors have nothing to disclose with regard to commercial support.

Received for publication Dec 9, 2011; revisions received Jan 25, 2012; accepted for publication March 12, 2012; available ahead of print May 24, 2012.

Address for reprints: Christine L. Lau, MD, Department of Surgery, University of Virginia Health System, PO Box 800679, Charlottesville, VA 22908-0679 (E-mail: cl12y@virginia.edu).

0022-5223/\$36.00

Copyright (C) 2013 by The American Association for Thoracic Surgery doi: $10.1016 /$ j.jtcvs.2012.03.079 inflammatory threats and microenvironmental cytokines. They travel to injured tissues by way of the CXCR4/ CXCL12 chemokine axis. Fibrocytes differentiate into $\alpha$-smooth muscle actin ( $\alpha$-SMA + ) producing fibroblasts/ myofibroblasts. ${ }^{2,3}$ Although fibrocytes have been shown to respond to several chemokines and express CCR3, CCR5, and CCR7 chemokines, the CXCR4/CXCL12 biologic axis is the predominate driving force for fibrocyte trafficking after airway injury. ${ }^{4,5}$

Fibrocytes were first described in the context of normal wound healing; however, their importance in the progression of fibrotic and asthmatic lung disease has since been demonstrated. ${ }^{6}$ Patients with exacerbation of idiopathic pulmonary fibrosis (IPF) have been shown to have an elevated proportion of peripheral blood fibrocytes compared with patients with stable IPF. Furthermore, the total circulating fibrocyte population in those with IPF was found to be an independent predictor of death. ${ }^{7}$

In a prospective study aimed at quantifying circulating fibrocyte populations (CD45+Col1+) in patients after lung transplantation, we found a statistically significant increase in the circulating fibrocyte number in patients diagnosed with BOS (defined according to the forced expiratory 


\section{Abbreviations and Acronyms \\ $\alpha$-SMA $=\alpha$-smooth muscle actin \\ BOS = bronchiolitis obliterans syndrome \\ Col1 = collagen 1 \\ IPF = idiopathic pulmonary fibrosis \\ mTOR $=$ mammalian target of rapamycin}

volume in 1 second) compared with that in patients without BOS $\left(8.9 \times 10^{5}\right.$ cells $/ \mathrm{mL}$ vs $2.96 \times 10^{5}$ cells $/ \mathrm{mL}$, respectively). Furthermore, we found significant incremental increases in circulating fibrocyte numbers with advancing BOS stage, suggesting a role for fibrocytes in BOS progression. ${ }^{8}$ This has been corroborated by another study, which found a statistically significant increase in the number of fibrocytes on staining for CXCR4/prolyl 4-hydroxylase in lung biopsy specimens from lung transplant patients with BOS compared with controls. ${ }^{9}$

The associations between fibrocyte numbers and BOS progression in human studies has suggested, but not yet proved, that fibrocytes have detrimental effects in pulmonary fibrosis. Given the correlation of fibrocyte number and clinical BOS stage and the importance of the CXCR4/CXCL12 axis in fibrocyte trafficking, we hypothesized that immunotherapy directed against CXCL12 would attenuate airway fibrosis and obliteration in a wellestablished murine heterotopic tracheal transplant model of obliterative bronchiolitis. ${ }^{10,11}$

\section{METHODS}

\section{Generation and Purification of Anti-CXCL12 IgG $\mathbf{F}\left(\mathbf{a b}^{\prime}\right)_{2}$ Fragments}

Goat anti-human CXCL12 antibodies were purified using the Melon Gel IgG purification Kit (Thermo Scientific, Rockford, Ill). Normal goat IgG and purified goat anti-human CXCL12 IgG were digested with Immobilized Pepsin (Pierce, Rockford, Ill). Fragments were recovered and dialyzed against phosphate-buffered saline. $\mathrm{F}\left(\mathrm{ab}^{\prime}\right)_{2}$ were purified from $\mathrm{Fc}$ fragments using NUNC Protein G columns (Nunc, Rockford, Ill).

\section{Mice}

All mice (Jackson Laboratory, Bar Harbor, Maine) received humane care according to the "Principles of Laboratory Animal Care," formulated by the National Society for Medical Research and the "Guide for the Care and Use of Laboratory Animals." The Animal Care and Use Committee at the University of Virginia approved the study protocol.

\section{Experimental Group Design}

We used a heterotopic subcutaneous tracheal transplant model of bronchiolitis obliterans, as previously described. ${ }^{11}$ A major histocompatability complex class I and II mismatch was produced by transplanting $4 \mathrm{Balb} / \mathrm{c}$ $\left(\mathrm{H}-2^{\mathrm{d}}\right)$ tracheas into $1 \mathrm{C} 57 \mathrm{BL} / 6\left(\mathrm{H}-2^{\mathrm{b}}\right)$ recipient. Four donor trachea allografts were used per recipient to ensure adequate tracheal tissue for fibrocyte determination and histologic examination. The mice were divided into 2 groups: the IgG control group [intraperitoneal injections of normal goat $\operatorname{IgG~F}\left(\mathrm{ab}^{\prime}\right)_{2}$ ] and the anti-CXCL12 group [intraperitoneal injections of antiCXCL12 $\mathrm{F}\left(\mathrm{ab}^{\prime}\right)_{2}$ ]. Each group included 6 recipients for a total of 120 donors and 30 recipients. Thus, there were a total of 240 donors and 60 recipients. All recipients were given intraperitoneal injections at days $-1,0$, and 1 , and then every other day until the endpoints. The mice were killed, and the allografts were collected on days $3,7,12,21$, or 28 . Tracheal allografts from 5 of the 6 mice at each point were pooled for flow cytometry analysis, and the tracheal allografts from the sixth mouse were used for histologic examination and Sirius Red staining.

\section{Fluorescence Activated Cell Sorter Analysis}

Single cell suspensions isolated from bone marrow, peripheral blood, and trachea allografts were stained with peridinin-chlorophyll-protein complex-labeled CD45, phycoerythrin-labeled CXCR4 (BD Biosciences, San Diego, Calif), or isotype controls. Subsequently, the cells were permeabilized using cytofix/cytoperm for staining with DyLight-488 conjugated anti-Col1, phycoerythrin-labeled $\alpha$-SMA, or isotype controls. Anti-Coll and a rabbit IgG isotype control were conjugated using a DyLight- 488 conjugation kit (Thermo Scientific, Rockford, Ill). Four-color analysis of the stained cells was performed on a FACSCanto II flow cytometer using FACSDiva, version 6.0, software (BD Biosciences). All analyses were blinded.

\section{Histologic Examination and Measurement of Fibrosis and Luminal Obliteration}

The allograft tracheal tissues were fixed, embedded, sectioned, and stained with hematoxylin and eosin. Allograft trachea sections were photographed at $4 \times$ magnification. Collagen deposition was quantified according to the percentage of luminal obliteration of the tracheal allografts using ImagePro Plus software. Eight allografts were measured in each group.

\section{Collagen Staining and Densitometry}

The tracheal sections were deparaffinized, rehydrated, and stained with Direct Red 80 (Sigma Aldrich, St. Louis, Mo). The images were captured for quantification using Image $\mathbf{J}$ software. The collagen signal in luminal fibro-obliteration tissue was semiquantified using the same software but with a different parameter setting.

\section{Statistical Analysis}

Fibrocytes from buffy coat, bone marrow, and tracheal single cell suspensions are presented as box and whisker plots. Each box and whisker plot at a given point represents 5 mice. Continuous data are reported as either the mean \pm standard deviation or median and interquartile range. Independent sample comparisons were performed using either the MannWhitney $U$ test or Kruskal-Wallis test. Statistical significance was set to $P<.05$. Group comparisons were unpaired, and the $P$ values are 2 -tailed. The analyses were performed using predictive analytics software (version 18.0.0; IBM Corp, Chicago, Ill).

\section{RESULTS}

\section{Attenuation of Fibrocyte Trafficking Using} Anti-CXCL12 F $\left(\mathbf{a b}^{\prime}\right)_{2}$ Antibody

We first assessed the ability of neutralizing anti-CXCL12 $\mathrm{F}\left(\mathrm{ab}^{\prime}\right)_{2}$ antibodies to attenuate fibrocyte trafficking and differentiation in the murine model of bronchiolitis obliterans. Bone marrow, buffy coat, and trachea allograft single-cell suspensions from Balb/C mice subcutaneously transplanted with BL6 trachea were analyzed for total undifferentiated (CD45+Col1+CXCR4+) and differentiated fibrocyte (CD45+Col1 $+\alpha-\mathrm{SMA}+$ ) cell populations.

The comparison of buffy coat isolates from mouse whole blood preparations revealed significant decrease in circulating undifferentiated fibrocytes in mice treated with 
anti-CXCL12 at 7 days (Figure $1, A ; P=.028$ ) after tracheal transplantation. However, anti-CXCL12-treated mice at 12 days after transplantation (Figure $1, A ; P=.028$ ) had increased undifferentiated fibrocytes compared with controls. Figure 1, $B$ shows a concomitant increase in total differentiated fibrocytes in buffy coat preparations from antiCXCL12-treated mice at 3 days $(P=.047)$, with a decrease in the differentiated fibrocyte population at 7 days $(P=.008)$.

Bone marrow aspirates showed an increase in undifferentiated fibrocytes (Figure 1, $C$ ) in anti-CXCL12-treated mice at 12 days after transplantation $(P=.015)$ and a decrease in undifferentiated fibrocytes at 7 days $(P=.008)$ and 21 days $(P=.014)$ after transplantation compared with the controls. Similarly, a significant increase was seen in the number of total differentiated fibrocytes (Figure $1, D$ ) in the antiCXCL12-treated mice at 12 days after transplantation $(P=.004)$, with a decrease in the same population at 7 days after transplantation $(P=.008)$.

As hypothesized, the single cell suspensions isolated from the tracheal allografts from anti-CXCL12-treated mice showed a significant decrease in both undifferentiated (Figure 1,E) and differentiated (Figure 1, F) fibrocytes at 7 days $(P=.009$ and $P=.009$, respectively) and 21 days $(P=.004$ and $P=.014$, respectively) after transplantation compared with the controls.

The mean ranks of undifferentiated and differentiated fibrocytes among buffy coat $(\mathrm{H}=18.95,7 \mathrm{df}, P=.008$ and $\mathrm{H}=21.92,7 \mathrm{df}, P=.003$, respectively), bone marrow $(\mathrm{H}=33.64,7 \mathrm{df}, P<.0001$ and $\mathrm{H}=34.01,7 \mathrm{df}$, $P<.0001$, respectively), and tracheal allograft single cell populations $(\mathrm{H}=30.29,7 \mathrm{df}, P<.0001$ and $\mathrm{H}=33.02,7$ df, $P<.0001$, respectively) were significantly different among the control and anti-CXCL12-treated mice. Thus, a definite variation in fibrocyte number was found within each group.

\section{Evaluation of Fibro-Obliteration on Tracheal Allografts}

Representative hematoxylin and eosin images of tracheal allografts from the anti-CXCL12 and control mice at 3, 7, 12 , and 21 days after transplantation are shown in Figure 2. Airway fibro-obliteration was scored using 2 methods. First, qualitative hematoxylin and eosin analysis was reviewed blindly by a lung pathologist who determined the percentage of luminal obliteration of tracheal allografts after tracheal transplantation (Figure 3). A significant decrease was seen in the median luminal obliteration at 21 days $(P=.010)$ in the anti-CXCL12-treated mice $(65 \%$ vs $100 \%$ obliterated in controls; interquartile range, $38 \%$ vs $10 \%)$. This trend was not seen at 28 days after transplantation $(P=.153)$.

We next assessed the effect of anti-CXCL12 treatment on collagen deposition in trachea allograft lumens. Densometric quantitative measurement of direct red 80 collagen staining was used to determine the total luminal collagen deposition during the same period. As expected, treatment with antiCXCL12 resulted in a significant decrease in trachea allograft luminal collagen deposition at 21 days (1591.62 vs 6314.97 integrated density units; interquartile range, 3167.98 vs $10,725.91 ; P=.042)$ and 28 days (6871.09 vs $12,775.80$ integrated density units; interquartile range, 5280.35 vs 8346.05; $P=.0012$ ) after transplantation (Figure 4). This is consistent with the hypothesis that inhibition of the CXCR4/CXCL12 biologic axis results in attenuated fibrocyte trafficking to, and differentiation in, tracheal allografts.

The kinetics of total nonluminal tracheal collagen deposition showed a slightly different pattern from that of luminal deposition (data not shown). A significant decrease in tracheal fibro-obliteration in the anti-CXCL12-treated mice at $12(P<.0001)$ and $21(P<.0001)$ days after transplantation compared with controls, with no significant difference seen at 3,7 , and 28 days $(P>.05)$.

\section{DISCUSSION}

The importance of fibrocyte biology has been established in multiple pancorporeal fibrotic processes, including normal wound repair, nephrogenic fibrosing dermatomyopathy, scleroderma, chronic pancreatitis, cystitis, liver fibrosis, and multiple lung pathologies marked by recurring inflammation and repair. ${ }^{6}$ We have previously shown a correlation between increased circulating fibrocyte levels and the development and progression of BOS in patients after lung transplantation. ${ }^{8}$ These studies have suggested that fibrocytes might contribute to the pathology of pulmonary fibrosis.

Given the potential clinical importance of fibrocytes in the progression of fibrotic pathology, we sought to identify the effect of an anti-CXCL12 $\mathrm{F}\left(\mathrm{ab}^{\prime}\right)_{2}$ antibody in mitigating tracheal fibro-obliteration. Despite its shortcomings, we used a murine tracheal transplant model of bronchiolitis obliterans, because it is well described and less technically demanding and time consuming compared with other models and reproducible in its production of obliterative airway disease. The critics of this model have noted that this is a large airway representation of small airway disease and that it fails to use a vascularized or aerated allograft. Other models of bronchiolitis obliterans have been developed, including the orthotopic tracheal model ${ }^{12}$; however, just as in the heterotopic model, this is a large airway model and is not vascularized. Furthermore, it is technically challenging and fails to uniformly develop histopathologic bronchiolitis obliterans. Similarly, single lung transplantation has been successfully performed in mice. However, these transplanted lungs allografts have not been shown to reliably develop histopathologic lesions of bronchiolitis obliterans. ${ }^{13}$

In the present study, we have demonstrated that an anti-CXCL12 antibody effectively attenuates fibrocyte 
$\mathrm{CD}^{+} 5^{+} \mathrm{Col}-1^{+} \mathrm{CXCR} 4^{+}$

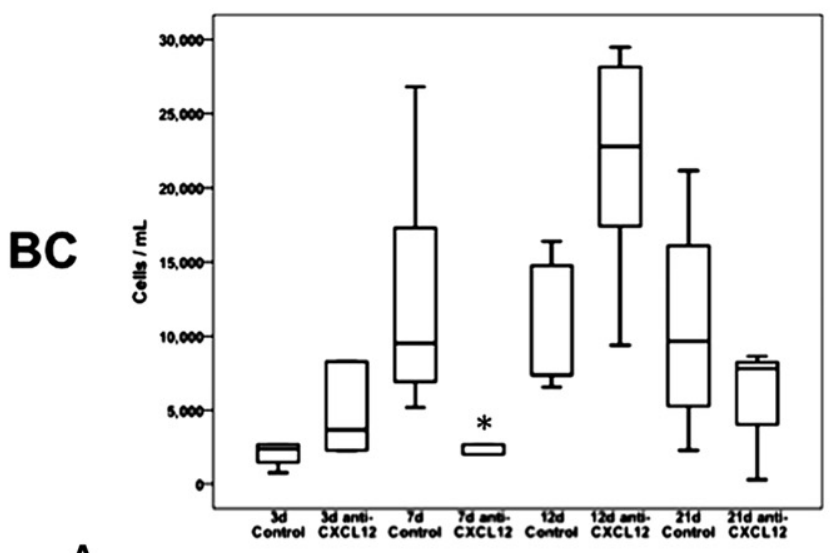

A

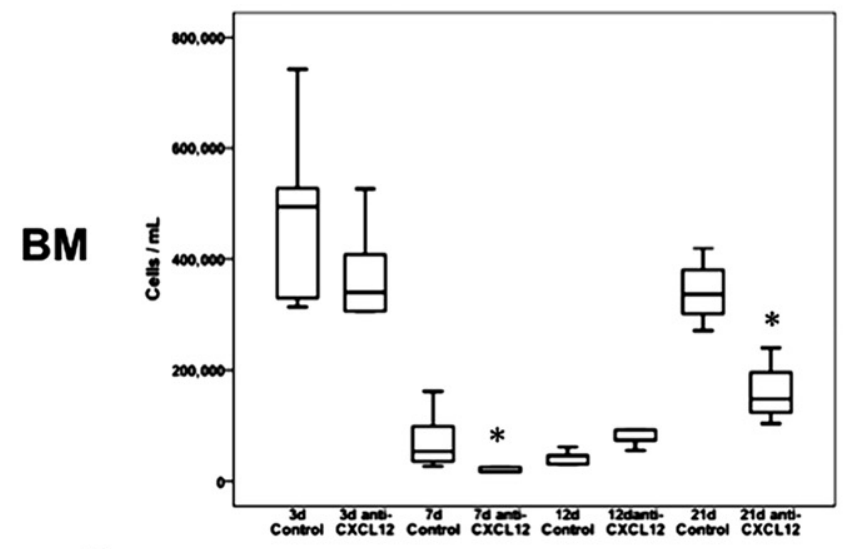

C

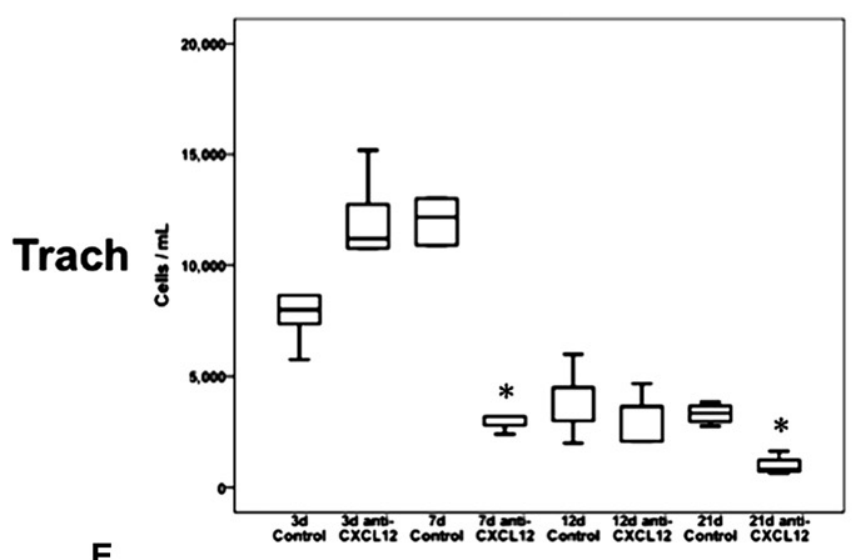

$\mathrm{CD}^{4} 5^{+} \mathrm{Col}-1^{+} \mathrm{SMA}^{+}$

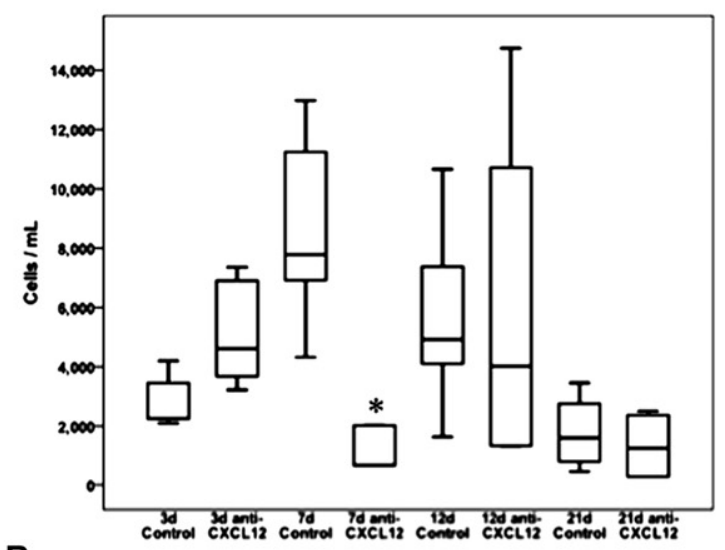

B

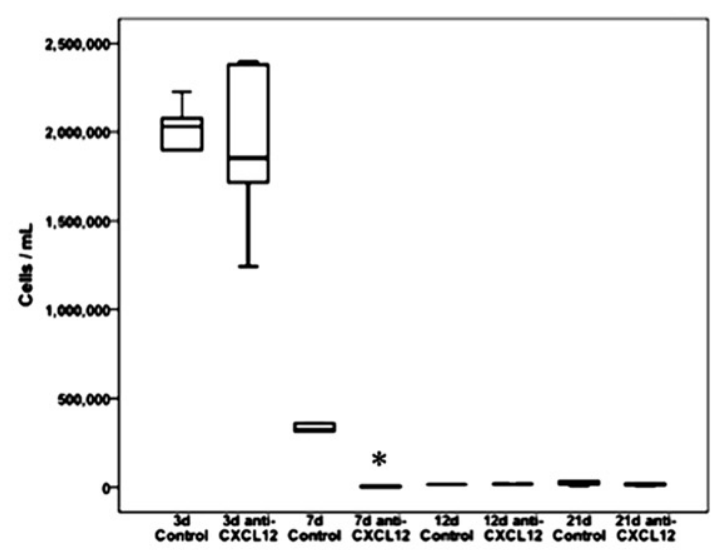

D

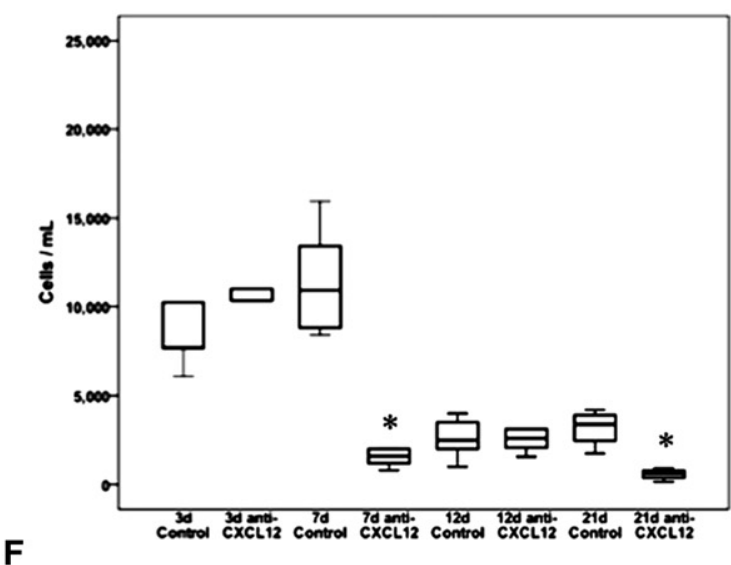

FIGURE 1. Box and whisker plots depicting total fibrocyte populations in both anti-CXCL12 $\mathrm{F}\left(\mathrm{ab}^{\prime}\right)_{2}$-treated and control goat IgG F(ab' $)_{2}-\operatorname{treated~mice~}$ after heterotopic transplant of allograft tracheas. Box and whiskers represent 25th to 75th and 10th to 90th percentiles, respectively; transverse lines represent the median. Each box and whisker plot at a given point represents 5 mice. A, C, E, Undifferentiated, CD45+collagen $1($ Coll) + CXCR4+fibrocyte populations over time in buffy coat $(B C)$, bone marrow $(B M)$, and tracheal allograft isolations (Trach), respectively. B, D, F, Differentiated, CD45+Col1+smooth muscle actin $(S M A)+$ fibrocyte populations in BC, bone marrow, and trachea allograft isolations, respectively. $* P<.05$, MannWhitney $U$ test. 


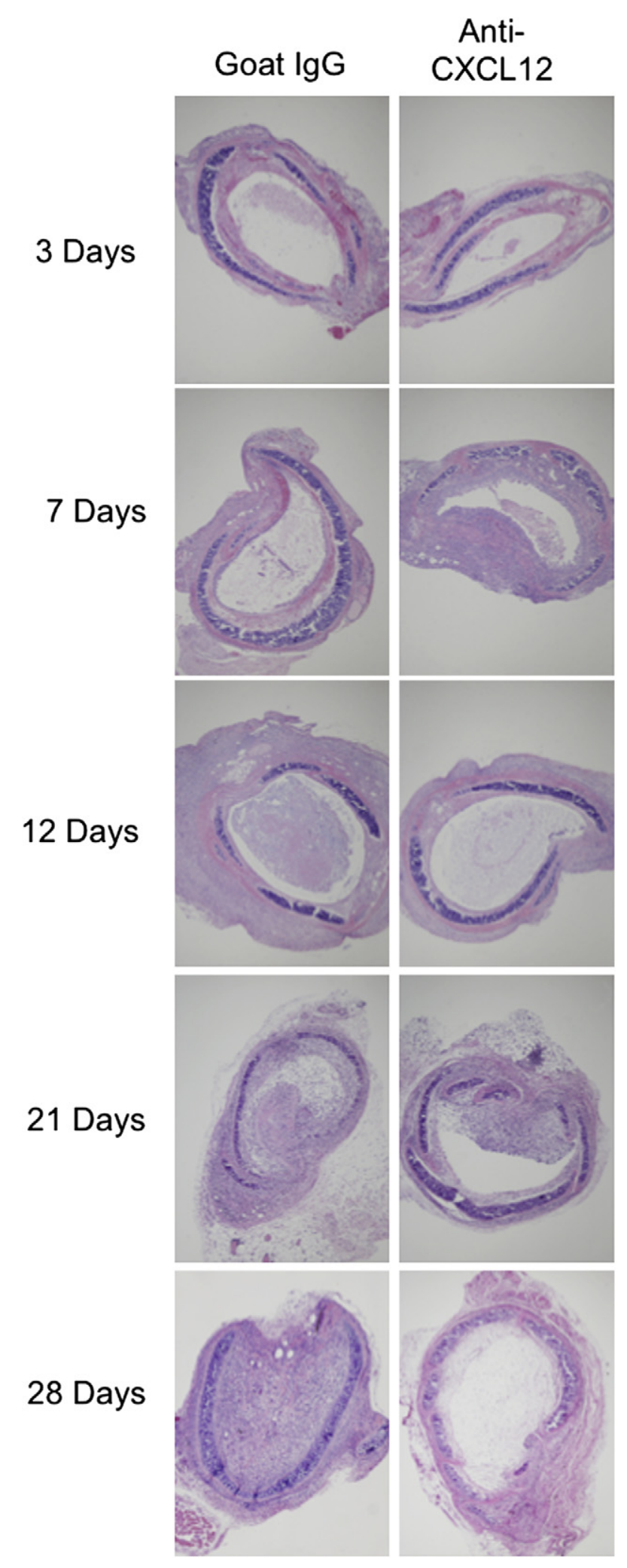

FIGURE 2. Representative images of hematoxylin and eosin-stained histopathologic sections of (left), goat $\operatorname{IgG~} \mathrm{F}\left(\mathrm{ab}^{\prime}\right)_{2}$-treated or (right), antiCXCL12 $\mathrm{F}\left(\mathrm{ab}^{\prime}\right)_{2}$-treated mice at 3, 7, 12, 21, and 28 days after heterotopic transplantation of allograft trachea. trafficking to, and differentiation in, heterotopic trachea allografts. This antibody simultaneously mitigated graft disease progression and fibro-obliteration. Our findings have been corroborated by evidence from other studies that found that blocking the CXCR4/CXCL12 axis with an anti-CXCL12 antibody $^{14}$ or a direct CXCR4 antagonist, AMD3100, ${ }^{15}$ protected against pulmonary fibrosis in bleomycin models of IPF - a process defined by cryptogenic fibro-obliteration of the lung parenchyma.

Recent evidence has pointed toward a duality in fibrocyte biology as a cell capable of both immune modulation and extracellular matrix repair. ${ }^{16}$ The differentiation of fibrocytes into cells capable of fulfilling these roles is dependent on the cytokine milieu and progresses chronologically during the disease course from an early role in an inflammatory phenotype to late differentiation into collagen-producing reparative cells. ${ }^{17}$ The results of the present study have shown a temporal fibrocyte trafficking pattern consistent with this paradigm in which fibrocytes respond to tracheal tissue injury by CXCL12 chemotaxis and propagate a temporal fibrotic process. In the acute stages after transplantation, there is an increase in bone marrow production of both undifferentiated (CD45+Col1+CXCR4+) and differentiated $(\mathrm{CD} 45+\mathrm{Col} 1+\alpha-\mathrm{SMA}+)$ fibrocytes in anti-CXCL12-treated and control mice. As expected, there was a lag in the appearance of both cell populations in buffy coat preparations.

As demonstrated in Figure 5, there is an early accumulation of undifferentiated fibrocytes in tracheal allografts that might result from the trafficking of fibrocytes already in circulation. However, there is a lack of early concomitant fibrosis, suggesting that these fibrocytes are responding to an inflammatory milieu that exists acutely after transplantation by differentiating along antigen-presenting or immunemodulatory cell lines instead of along a reparative cell lineage. A comparison of control and anti-CXCL12 mice demonstrated a more rapid and, ultimately, more significant decrease in fibrocyte numbers in anti-CXCL12-treated mice and a more rapid and, ultimately, more significant increase in tracheal fibrosis in the control mice. A KruskalWallis analysis demonstrated that both control and anti-CXCL12 fibrocyte populations $(P<.001)$ and tracheal collagen deposition $(P<.001)$ are significantly different.

On closer analysis, the difference between the control and anti-CXCL12-treated mice was most pronounced at day 7 , when the anti-CXCL12-treated mice have a strikingly decreased number of both fibrocyte populations in buffy coat and trachea allograft isolations. Although the control mice continued to experience an increase in fibrocyte trafficking and differentiation into grafted airways, both fibrocyte populations in the anti-CXCL12-treated mice greatly decreased. Therefore, day 7 might be significant in that it represents when the pressure to differentiate toward an inflammatory lineage subsides and the pressure to differentiate toward a reparative lineage increases. 


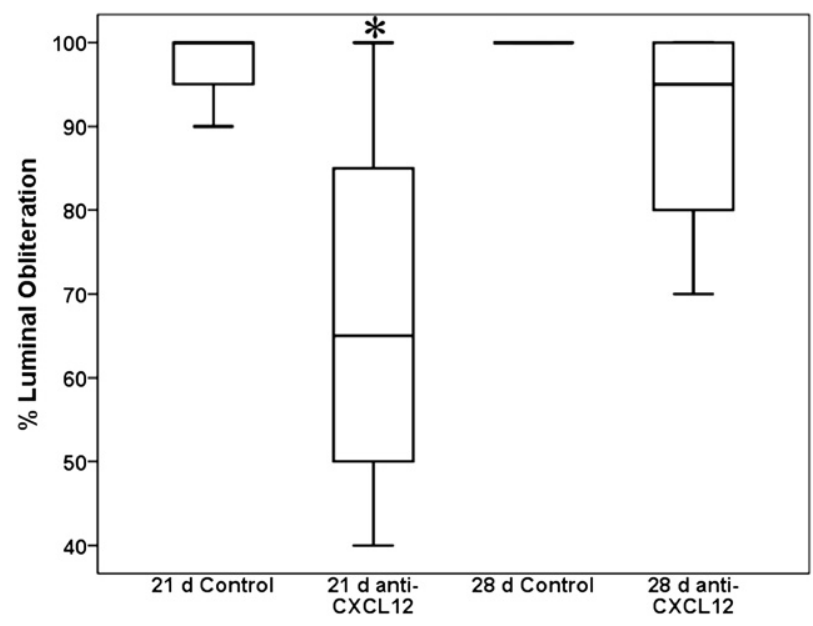

FIGURE 3. Box and whisker plots depicting percentage of luminal fibrosis in tracheal allograft sections in both anti-CXCL12 $\mathrm{F}\left(\mathrm{ab}^{\prime}\right)_{2}$-treated and control goat IgG F( $\left.\mathrm{ab}^{\prime}\right)_{2}$-treated mice as assessed by a lung pathologist in a blinded fashion. Box and whiskers represent 25 th to 75 th and 10th to 90th percentiles, respectively; transverse lines represent the median. ${ }^{*} P<.05$, Mann-Whitney $U$ test.

Thus, the larger fibrocyte populations on day 7 in the control mice are able to respond to this pressure and, as a result, fibro-obliteration occurs more rapidly than in the antiCXCL12-treated mice.

Our data have shown that inhibiting the CXCL12/ CXCR4 axis had little effect on the acute bone marrow production of fibrocytes but instead appeared more vital in preventing the trafficking of fibrocytes from the bone marrow into the circulation and from the circulation into the extravascular compartment. Furthermore, the difference in kinetics of collagen deposition between tracheal tissue and

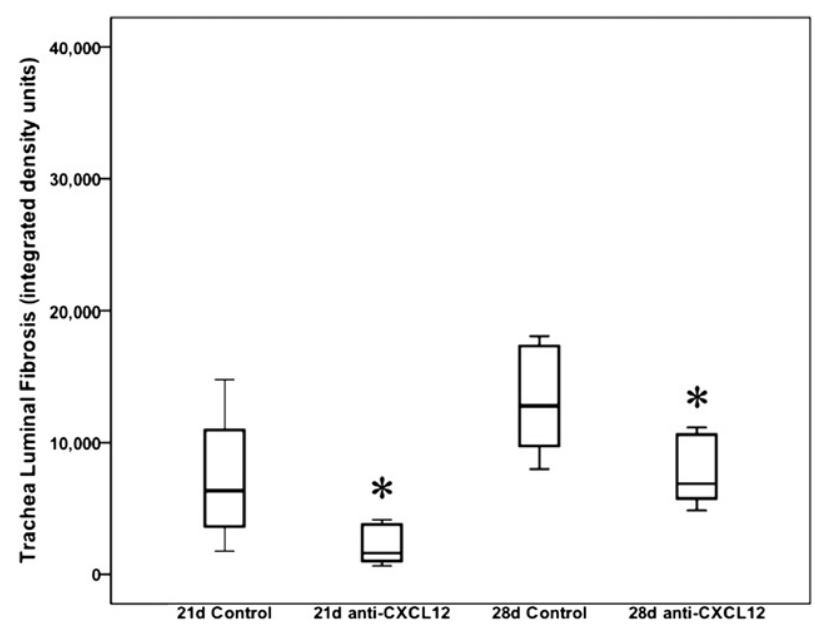

FIGURE 4. Box and whisker plots depicting percentage of tracheal luminal fibrosis as measured using Direct Red 80 collagen stain at 21 and 28 days after transplantation. Box and whiskers represent 25th to 75 th and 10th to 90th percentiles, respectively; transverse lines represent the median. ${ }^{*} P<.05$, Mann-Whitney $U$ test. lumen implicates the CXCL12/CXCR4 axis in the trafficking of fibrocytes through the tracheal tissue and into the tracheal lumen. At day 28, no difference was seen in tracheal tissue collagen deposition between the anti-CXCL12treated mice and controls; however, the treated mice showed a significant decrease in total luminal collagen deposition, indicating that although the anti-CXCL12 antibody did not completely prevent the fibrotic disease from progressing in the tracheal allograft tissue, it mitigated total airway obliteration.

The inhibition of CXCL12 could be responsible for the decreased population of differentiated fibrocytes in the trachea allografts and buffy coat isolations and the decrease in luminal fibrosis in the anti-CXCL12-treated mice. However, it is likely that these changes were, in large part, a consequence of diminished trafficking and extravasation of undifferentiated fibrocytes into the proper milieu for differentiation. Although a drastic decrease was seen in both buffy coat and tracheal undifferentiated and differentiated fibrocyte populations in the anti-CXCL12-treated mice, there was a substantial increase in the same populations in the control mice. This might suggest that in the control mice, undifferentiated fibrocytes were more readily able to traffic to the tracheal allografts and differentiate into fibroblastic, collagen-producing cells. Although the difference in fibrocytes populations might not account for the entire difference in fibro-obliteration between the antiCXCL12-treated and control mice, it does seem to be a major contributor to disease progression.

However, we still saw an increasing accumulation of collagen deposition in the tracheal allografts from the antiCXCL12-treated mice. This might have been due, in part, to other less important chemokines (CCR7/CCR2) that are able to recruit fibrocytes into the allografts. Mouse fibrocytes have been shown to express CCR7, CXCR4, and CCR2 and respond to a number of different ligands. ${ }^{18}$ The CXCR4/CXCL12 axis is known to be the predominant receptor/ligand interaction for trafficking to a grafted airway and subsequent extravasation of fibrocytes into tissue. .,18,19 $^{-19}$ These other receptor/ligand interactions might help to explain the differences in kinetics between the antiCXCL12-treated and control groups. Furthermore, local differentiation of fibroblasts and epithelial to mesenchyme transition play a role in fibro-obliteration. ${ }^{2}$ The stimuli for fibrocyte production in the bone marrow and subsequent movement to blood is not clear, but it appears that other cytokine/receptor interactions might predominate. Thus, blocking the CXCR4/CXCL12 axis, although instrumental for inhibiting the progression of fibro-obliteration, does not, by itself, eliminate collagen deposition.

Our studies have provided novel insight into the kinetics of fibrocyte trafficking during the progression of airway fibro-obliteration and demonstrate the potential that inhibiting this axis has for slowing the disease process. 

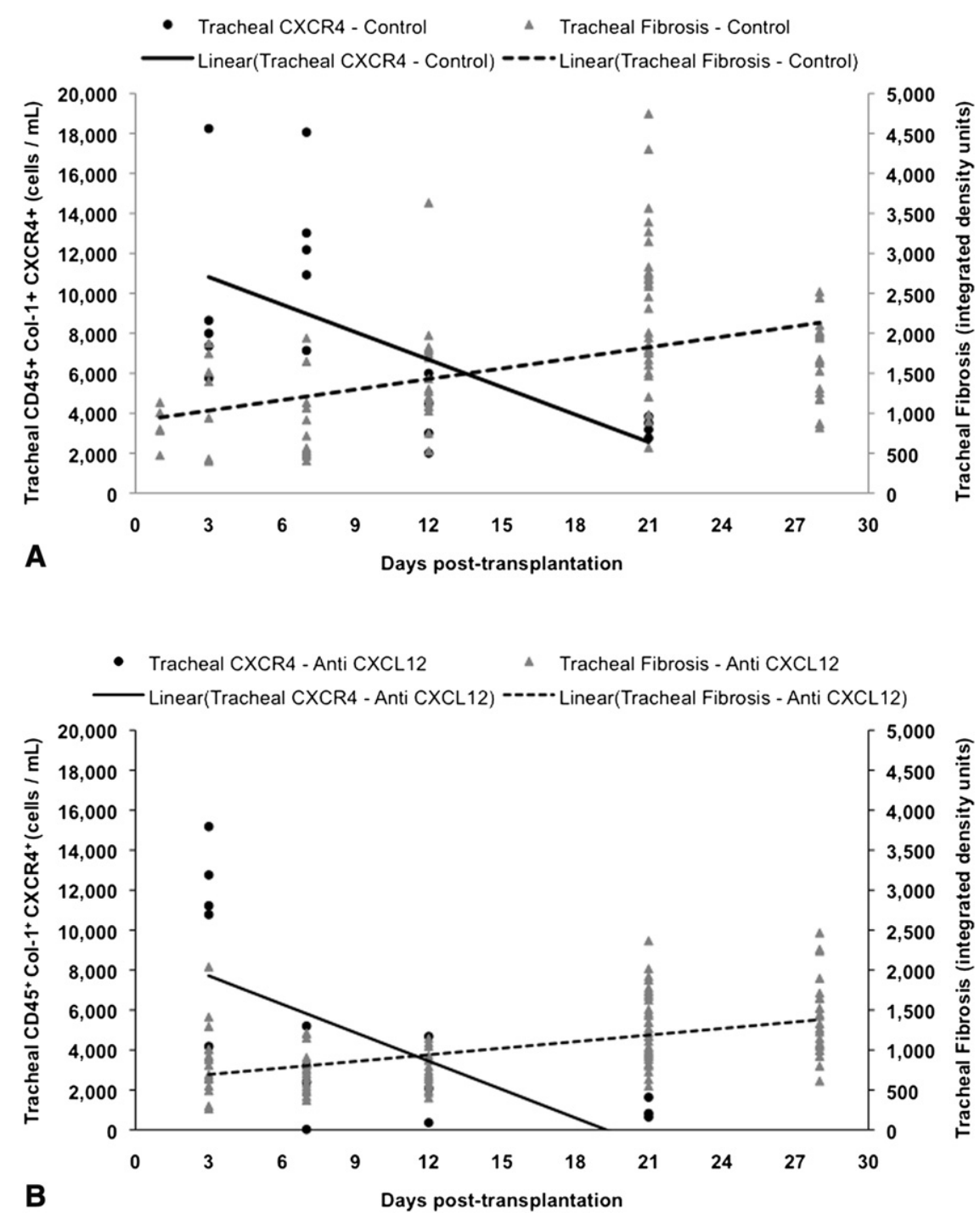

FIGURE 5. Combined kinetics of both undifferentiated, CD45+collagen 1 (Coll) + CXCR4+fibrocytes in tracheal allografts, as determined by flow cytometry, and tracheal tissue collagen deposition, as determined by Direct Red 80 densitometry of tracheal allograft sections. A, Kinetics of control, goat IgG $\mathrm{F}\left(\mathrm{ab}^{\prime}\right)_{2}$-treated mice showed more gradual and less complete decrease in tracheal allograft undifferentiated fibrocyte number with a concomitant more rapid and greater increase in tracheal collagen deposition compared with (B) anti-CXCL12 $\mathrm{F}\left(\mathrm{ab}^{\prime}\right)_{2}$-treated mice. Kruskal-Wallis analysis demonstrated that both control and anti-CXCL12 fibrocyte populations $(P<.001)$ and tracheal collagen deposition $(P<.001)$ were significantly different.

Anti-CXCL12 $\mathrm{F}\left(\mathrm{ab}^{\prime}\right)_{2}$ afforded protection against infiltrating fibrocytes and deterioration of allograft tracheal epithelium and collagen deposition. Thus, the CXCL12/CXCR4 axis provides a novel therapeutic target for the treatment of fibroproliferative disease. Given the clinical effect that fibrotic diseases have on morbidity and mortality, additional study is warranted in both animal models and humans to identify the role of this axis in molding fibrocyte differentiation in inflammatory and reparative milieus.

Newer immunomodulatory drugs directed toward inhibition of the mammalian target of rapamycin (mTOR), such as sirolimus and everolimus, offer significant hope in the prevention of allograft rejection. ${ }^{20}$ Previous studies have shown that therapy with rapamycin attenuates fibrocyte trafficking from bone marrow and prevents fibrocyte and collagen deposition in lungs challenged with bleomycin by altering CXCR4 expression. ${ }^{5}$ Others have shown everolimus to be a potent inhibitor of proliferation of ex vivo lung fibroblasts cultured from transbronchial biopsy samples harvested from patients after lung transplantation. ${ }^{21}$ Also, in 2 separate randomized control trials of lung transplant recipients, those patients receiving maintenance everolimus were less likely to have clinically suspected and biopsy proven acute graft rejection compared with the controls. ${ }^{22,23}$ Because these drugs are currently approved by the Food and Drug Administration for the prevention of 
solid organ rejection and their potency for immune modulation, they might prove to be superior alternatives to anti-CXCL12 $\mathrm{F}\left(\mathrm{ab}^{\prime}\right)_{2}$. Despite this potential, many therapeutic challenges remain to the proper administration of these drugs. In lung transplant recipients receiving sirolimus, an increased risk of severe venous thromboembolism was present,${ }^{20}$ and in separate trials, patients treated with sirolimus experienced airway anastomosis dehiscence and generalized poor wound healing. ${ }^{24,25}$ Thus, many clinicians have decided to administer sirolimus after completion of bronchiolar wound healing, ${ }^{24}$ and, as such, the use of sirolimus across all centers was $8 \%$ at 1 year and $18 \%$ at 5 years after transplantation. ${ }^{1}$

\section{CONCLUSIONS}

An optimal outcome of mTOR inhibition would allow mitigation of fibroproliferative disease and, at the same time, normal wound repair. We suggest that given the integral nature of mTOR and the CXCR4 biologic axis to the pathogenesis of fibrosis, monitoring fibrocyte populations could offer a new biomarker for optimizing mTOR inhibitor therapy. Quantification of the circulating and lung allograft fibrocyte populations might provide the clinician with an individualized view of fibrotic stage/progression in lung transplant patients. Using these populations as biomarkers, it might be possible to tailor mTOR inhibitor drug therapy to a dose that would both maximize gain and minimize unwanted side effects on an individual basis, effectively opening the door to a more personalized medical approach for these patients.

\section{References}

1. Christie JD, Edwards LB, Kucheryavaya AY, Benden C, Dobbels F, Kirk R, et al. The Registry of the International Society for Heart and Lung Transplantation: Twenty-Eighth Adult Lung and Heart-Lung Transplant Report-2011. J Heart Lung Transplant. 2011;30:1104-22.

2. Strieter RM, Mehrad B. New mechanisms of pulmonary fibrosis. Chest. 2009; 136:1364-70

3. Strieter RM, Keeley EC, Burdick MD, Mehrad B. The role of circulating mesenchymal progenitor cells, fibrocytes, in promoting pulmonary fibrosis. Trans Am Clin Climatol Assoc. 2009;120:49-59.

4. Strieter RM, Gomperts BN, Keane MP. The role of CXC chemokines in pulmonary fibrosis. J Clin Invest. 2007;117:549-56.

5. Mehrad B, Burdick MD, Strieter RM. Fibrocyte CXCR4 regulation as a therapeutic target in pulmonary fibrosis. Int J Biochem Cell Biol. 2009;41:1708-18.

6. Herzog EL, Bucala R. Fibrocytes in health and disease. Exp Hematol. 2010;38: 548-56.
7. Moeller A, Gilpin SE, Ask K, Cox G, Cook D, Gauldie J, et al. Circulating fibrocytes are an indicator of poor prognosis in idiopathic pulmonary fibrosis. Am J Respir Crit Care Med. 2009;179:588-94.

8. Lapar DJ, Burdick MD, Emaminia A, Harris DA, Strieter BA, Liu L, et al. Circulating fibrocytes correlate with bronchiolitis obliterans syndrome development after lung transplantation: a novel clinical biomarker. Ann Thorac Surg. 2011;92: 470-7.

9. Andersson-Sjöland A, Erjefält JS, Bjermer L, Eriksson L, WestergrenThorsson G. Fibrocytes are associated with vascular and parenchymal remodelling in patients with obliterative bronchiolitis. Respir Res. 2009;10:103.

10. Zhao Y, Lapar DJ, Steidle J, Emaminia A, Kron IL, Ailawadi G, et al. Adenosine signaling via the adenosine $2 \mathrm{~B}$ receptor is involved in bronchiolitis obliterans development. J Heart Lung Transplant. 2010;29:1405-14.

11. Lau CL, Zhao Y, Kron IL, Stoler MH, Laubach VE, Ailawadi G, et al. The role of adenosine A2A receptor signaling in bronchiolitis obliterans. Ann Thorac Surg. 2009;88:1071-8.

12. Okazaki M, Krupnick AS, Kornfeld CG, Lai JM, Ritter JH, Richardson SB, et al. A mouse model of orthotopic vascularized aerated lung transplantation. Am J Transplant. 2007;7:1672-9.

13. Hele DJ, Yacoub MH, Belvisi MG. The heterotopic tracheal allograft as an animal model of obliterative bronchiolitis. Respir Res. 2001;2:169-83.

14. Phillips RJ, Burdick MD, Hong K, Lutz MA, Murray LA, Xue YY, et al. Circulating fibrocytes traffic to the lungs in response to CXCL12 and mediate fibrosis. J Clin Invest. 2004;114:438-46.

15. Song JS, Kang CM, Kang HH, Yoon HK, Kim YK, Kim KH, et al. Inhibitory effect of CXC chemokine receptor 4 antagonist AMD3100 on bleomycin induced murine pulmonary fibrosis. Exp Mol Med. 2010;42:465-72.

16. Balmelli C, Alves MP, Steiner E, Zingg D, Peduto N, Ruggli N, et al. Responsiveness of fibrocytes to toll-like receptor danger signals. Immunobiology. 2007;212 693-9.

17. Reilkoff RA, Bucala R, Herzog EL. Fibrocytes: emerging effector cells in chronic inflammation. Nat Rev Immunol. 2011;11:427-35.

18. Gomperts BN, Strieter RM. Fibrocytes in lung disease. J Leukoc Biol. 2007;82 449-56.

19. Xu J, Mora A, Shim H, Stecenko A, Brigham KL, Rojas M. Role of the SDF-1/ CXCR4 axis in the pathogenesis of lung injury and fibrosis. Am J Respir Cell Mol Biol. 2007;37:291-9.

20. Ahya VN, McShane PJ, Baz MA, Valentine VG, Arcasoy SM, Love RB, et al Increased risk of venous thromboembolism with a sirolimus-based immunosuppression regimen in lung transplantation. J Heart Lung Transplant. 2011;30 175-81.

21. Azzola A, Havryk A, Chhajed P, Hostettler K, Black J, Johnson P, et al. Everolimus and mycophenolate mofetil are potent inhibitors of fibroblast proliferation after lung transplantation. Transplantation. 2004;77:275-80.

22. Kovarik JM, Snell GI, Valentine V, Aris R, Chan CKN, Schmidli H, et al. Everolimus in pulmonary transplantation: pharmacokinetics and exposure-response relationships. J Heart Lung Transplant. 2006;25:440-6.

23. Snell GI, Valentine VG, Vitulo P, Glanville AR, McGiffin DC, Loyd JE, et al Everolimus versus azathioprine in maintenance lung transplant recipients: an in ternational, randomized, double-blind clinical trial. Am J Transplant. 2006;6: 169-77.

24. Groetzner J, Kur F, Spelsberg F, Behr J, Frey L, Bittmann I, et al. Airway anastomosis complications in de novo lung transplantation with sirolimus-based immunosuppression. J Heart Lung Transplant. 2004;23:632-8.

25. King-Biggs MB, Dunitz JM, Park SJ, Kay Savik S, Hertz MI. Airway anastomotic dehiscence associated with use of sirolimus immediately after lung transplantation. Transplantation. 2003;75:1437-43. 